\title{
Neuroscience \\ Impaired sequential but preserved motor memory consolidation in Multiple Sclerosis disease \\ --Manuscript Draft--
}

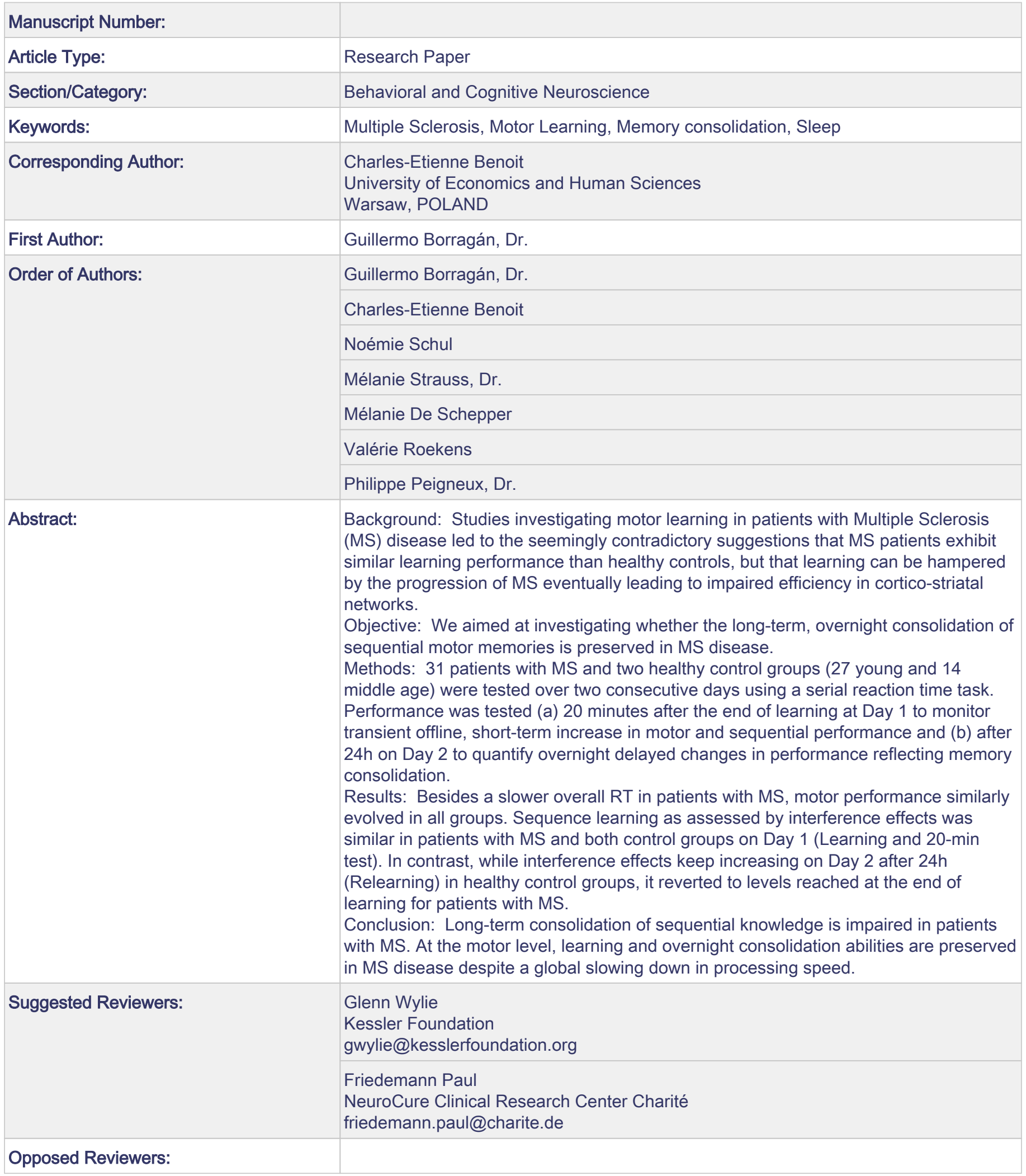




\title{
Impaired sequential but preserved motor memory consolidation in Multiple \\ Sclerosis disease
}

\author{
Guillermo Borragán*1 and Charles-Etienne Benoit*+2 ${ }^{2}$, Noémie Schul ${ }^{1}$, Mélanie Strauss ${ }^{1,3}$, Mélanie De \\ Schepper ${ }^{1}$, Valérie Roekens ${ }^{4}$ and Philippe Peigneux ${ }^{1}$ \\ * Co-first authors \\ †Corresponding author: c.benoit@vizja.pl \\ ${ }^{1}$ UR2NF, Neuropsychology and Functional Neuroimaging Research Unit at CRCN, Centre de Recherches en \\ Cognition et Neurosciences and UNI - ULB Neurosciences Institute, Université Libre de Bruxelles (ULB), \\ Belgium \\ ${ }^{2}$ Faculty of Psychology, University of Economics and Human Sciences in Warsaw, Warsaw 01-043, Poland \\ ${ }^{3}$ Neurology and Psychiatry \& Sleep Departments, Cliniques Universitaires de Bruxelles - Hôpital Erasme, \\ Université Libre de Bruxelles (ULB), Belgium \\ ${ }^{4}$ Nationaal Multiple Sclerose Centrum vzw, Melsbroek, Belgium
}

\section{Word counts:}

3056

\section{Keywords:}

Multiple Sclerosis, Motor Learning, Memory consolidation, Sleep 


\begin{abstract}
:
Background: Studies investigating motor learning in patients with Multiple Sclerosis (MS) disease led to the seemingly contradictory suggestions that MS patients exhibit similar learning performance than healthy controls, but that learning can be hampered by the progression of MS eventually leading to impaired efficiency in cortico-striatal networks.
\end{abstract}

Objective: We aimed at investigating whether the long-term, overnight consolidation of sequential motor memories is preserved in MS disease.

Methods: 31 patients with MS and two healthy control groups (27 young and 14 middle age) were tested over two consecutive days using a serial reaction time task. Performance was tested (a) 20 minutes after the end of learning at Day 1 to monitor transient offline, short-term increase in motor and sequential performance and (b) after $24 \mathrm{~h}$ on Day 2 to quantify overnight delayed changes in performance reflecting memory consolidation.

Results: Besides a slower overall RT in patients with MS, motor performance similarly evolved in all groups. Sequence learning as assessed by interference effects was similar in patients with MS and both control groups on Day 1 (Learning and 20-min test). In contrast, while interference effects keep increasing on Day 2 after 24h (Relearning) in healthy control groups, it reverted to levels reached at the end of learning for patients with MS.

Conclusion: Long-term consolidation of sequential knowledge is impaired in patients with MS. At the motor level, learning and overnight consolidation abilities are preserved in MS disease despite a global slowing down in processing speed. 


\section{INTRODUCTION:}

Multiple sclerosis (MS) disease is a chronic inflammatory autoimmune pathology of the central nervous system. MS eventually results in neuronal damage both in grey and white matter, ultimately decreasing the efficiency of neural transmission. MS symptoms are various and can affect sensory, motor and/or cognitive domains, including mental fatigue (1). In particular, motor skill learning capabilities may become limited with the progression of the disease due to increased sensory and motor deficits (2) associated with alterations in cortico-striatal networks (3). However, report of preserved abilities to acquire new motor skills even at an advanced stage (4) may contradict this assumption. Additionally, MS is associated with decreased sleep quality and organization (5) that may in turn exert a deleterious impact on brain functioning and associated brain plasticity processes.

The temporal dynamics of motor skill learning are a topical focus of research (6). In healthy participants, motor skills develop over successive steps both online, i.e., during actual motor learning, and offline, i.e., in the absence of actual practice during post-training periods. Fast and slow experience-driven changes, reflected in underlying neural structures, parallel this development (7). During motor learning, performance rapidly increases with practice. Offline, it continues spontaneously improving in a multi-step, dynamic process. In healthy young participants, motor performance strikingly improves within the first 5 to 30 minutes after the end of practice (8-11) as well as at delayed testing $24 \mathrm{~h}$ to $48 \mathrm{~h}$ later (8-10). At variance, performance remains at the level reached at the end of the learning session when tested 4-5 hours after learning (8-10). The 30-minutes and 4-hours post-training testing phases are coined boost and silent periods (7), respectively. Performance levels reached at the boost phase were found predictive of delayed performance $48 \mathrm{~h}$ later, suggesting the potential relevance of immediate post-training periods for the development of longer-term memory consolidation processes. Additionally, post-training sleep was shown beneficial for the offline improvement of sequential motor skills (11), partly in relation to sleep spindles activity during NREM2 sleep (for a review, see e.g. (12)). 
The present study aimed at characterizing the temporal dynamics of the acquisition and consolidation of a sequential motor skill in patients with MS disease and healthy controls 20minutes and 24-hours post-training, in relation with reported sleep quality.

\section{METHODS}

\subsection{Participants}

Thirty-one patients diagnosed with MS (average \pm std age $=44.5 \pm 14.2$ years) and 41 healthy controls (Table 1) gave written agreement to participate in this study approved by National MS Center Melsbroek Ethics Committee. They were recruited locally from the Center Melsbroek, but also from Arlon's hospital in the south of Belgium. To control for a possible effect of age in MS (13), 14 middle-age (Healthy Middle; Age = 53.1 \pm 5.8 yrs) and 27 young-age (Healthy Young; Age $=21 \pm 1.2 \mathrm{yrs}$ ) adults with no history of neurological, psychiatric condition or sleeping disorder constituted the control population. Exclusion criteria for MS patients were documented cognitive decline, anxiety or depressive symptoms, and/or major motricity loss that would prevent performing the motor leaning task.

\subsection{Material}

\subsubsection{Sleep and Fatigue}

Participants completed the Pittsburgh Sleep Quality Index - PSQI (14) and the Fatigue Scale for Motor and Cognitive functions- FSMC (15) to obtain information about habitual sleep quality (PSQI) and cognitive, physical and social dimensions of fatigue (FSMC) over the last month. MS patients additionally completed the Expanded Disability Status Scale - EDSS (16) to determine their degree of disability in the motor domain. For the two nights prior and post motor learning (Figure 1b), quantitative (sleep duration, time spent in bed) and qualitative (quality of sleep, alertness at awakening) sleep measures were obtained using the self-reported St-Mary Hospital Sleep Questionnaire (QSN) (17). 


\subsubsection{Motor learning task}

We used a touchscreen (Magic Touch Add-On Touch Screen, KeyTec-Inc.) variant of the Serial Reaction Time (SRT) task (for a detailed presentation, see Borragán et al., 2015 (11)). Participants were instructed to respond as quickly and accurately as possible by pressing with the non-dominant hand on the stimulus (i.e., a car on a race circuit) presented at one out of four possible $5 \times 6 \mathrm{~cm}$ squares, located at each corner of the screen (Figure 1a). The stimulus remained on screen until the subject's response, then the next one was displayed immediately after (response stimulus interval [RSI] $0 \mathrm{~ms}$ ). Unbeknownst to participants, the succession of locations within each 64-trials block was either random (R) or repeated 8 times a fixed 8-elements sequence (S; L1 [ $\left.\begin{array}{llllllll}1 & 3 & 4 & 2 & 3 & 1 & 2 & 4\end{array}\right]$ or L2 [ [ [ $\left.\begin{array}{lllllll}4 & 1 & 3 & 2 & 4 & 3 & 1\end{array}\right]$ ).

\subsection{Procedure}

On Day 1 (Figure 1b), participants first practiced the SRT task for nine blocks (learning session) using one of the two fixed sequences L1 or L2, counterbalanced between participants. Stimuli were presented following the repeated sequence in blocks S2 to S6 and S8-S9, and randomized in Blocks R1, R7. After a 20-minutes break (Retest - 20 min session), participants performed the SRT task again for 4 additional blocks alternating the learned repeated (S10-S11, S13) and the random (R12) blocks. On the next day after a regular night of sleep at home (Retest - 24h session; Figure 1c), they were tested again for 4 blocks using the same setting (S14-S15, R16, S17). Participants were always tested at the same time of the day to avoid circadian confounds. SRT practice during postlunch dip (13-14h) was avoided (18).

\section{INSERT FIGURE 1}

\subsection{Data Availability}

The authors take full responsibility for the integrity of data and agree to share any data not published within this article upon reasonable request from any qualified investigator. Raw anonymized data will be made available on Dryad upon publication. 


\section{RESULTS}

\subsection{Age, Sleep and Fatigue}

One MS patient was excluded from the sample due to outlier performance during motor learning ( $\sum$ of $R T s>2$ std). Patients with MS differed from both control groups according to age (One-way ANOVA $F_{(2,68)}=66.0 ; p<.001$ ), being on average younger than Healthy Middle (Tukey posthoc $p<.05$ ) and older than Healthy Young (post-hoc $p<.0 .01$ ) controls. A one-way ANOVA conducted on global PSQI scores with between-subject factor Group (MS vs. Healthy Middle vs. Healthy Young) revealed a main Group effect $\left(F_{(2,68)}=10.1 ; p<.001 ; M S E=10.3 ;\right.$ partial $-\eta^{2}=$ $.23)$, with higher PSQI scores (i.e., lower sleep quality) in MS patients $(8.9 \pm 4.3 ; \mathrm{p}<0.01)$ than healthy controls (Middle-Age $5.3 \pm 2=$ Young $5.4 \pm 2.2 ; \mathrm{p}>.9$; see Table 1).

Additionally, Pearson correlations investigated separately in MS patients the potential relationship between sleep quality (PSQI), disability status (EDSS score) and the feeling of mental fatigue (FSMC-cognitive). PSQI and FSMC-cognitive scores were positively correlated $(\mathrm{r}=.38, p<.05)$, suggesting that patients with poor sleep experience higher daily mental fatigue. No relationship was found between PSQI and EDSS scores $(\mathrm{p}>.4)$.

\section{INSERT TABLE 1}

\subsection{SRT task}

Mean reaction times (RTs) for correct responses were computed for each block separately (Figure 2). Since accuracy (defined as responses given within the screen area in which the stimulus was presented) was close to ceiling in all groups ( $>99 \%$ ), analyses were conducted on speed measures only after removal of outliers (RTs $>2$ Std from the mean). Evolution of RTs between blocks was normally distributed in all groups (Anderson-darling normality tests $p s>.5$ ). Besides motor speed reflected by mean RTs, the learning of the sequential regularities was assessed computing at each session an Interference index, i.e., the percentage of increase in RTs prompted by the inclusion of a random block (BR) as compared to the two adjacent sequential (S) Badj and Badj $_{2}$ blocks: 


$$
\text { Interference index }=\left[B R-\frac{\left(B a d j_{1}+B a d j_{2}\right) / 2}{B R}\right] * 100
$$

Also, as they have been shown different between groups (see above and Table 1), PSQI scores and Age were systematically introduced as covariates in the ANOVAs reported hereafter.

\subsubsection{Motor performance: Day 1 learning session}

First, we evaluated motor learning abilities (independently of sequential knowledge) as the evolution of mean RTs within the first 6 blocks at Day 1. Random block R1 was included in this analysis as it provides a baseline measure for motor performance. A repeated-measure ANOVA on RTs with within-subject factor Block (6 levels: R1-S6) and between-subject factor Group (MS vs. Healthy Middle vs. Healthy Young) disclosed a main Group effect $\left(F_{(2,66)}=19.8 ; p<.001 ; M S E=\right.$ 124488; partial $-\eta^{2}=.37$ ). Post-hoc tests indicate slower RTs in patients with MS than in healthy controls [MS: $889 \pm 167 \mathrm{msec}>$ (Healthy Middle: $655 \pm 244=$ Healthy Young : 547 \pm 176 ); $p s<$

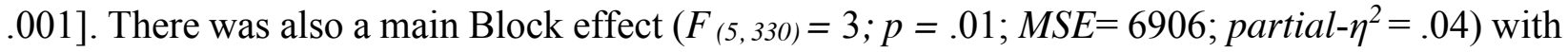
progressively faster RTs [Tukey post-hoc $\mathrm{R} 1>\mathrm{S} 2>\mathrm{S} 4>\mathrm{S} 6$; all $p s<.05$ ]. The Age covariate significantly modulated speed in all groups $\left(F_{(1,66)}=25 ; p=.001 ; M S E=124488 ;\right.$ partial $\left.-\eta^{2}=.28\right)$ but did not interact with performance evolution $(\mathrm{p}>.24)$. Finally, the Group $\times$ Block interaction did not reach significance $(F<.8, p>.55)$, suggesting that besides a slower overall RT in patients with MS, motor performance similarly evolved in all groups.

\section{INSERT FIGURE 2}

\subsubsection{Offline evolution of motor performance}

We computed a repeated measures ANOVA on mean RTs changes (i.e., last block of one session vs. first block of the next one) with between-subject factor Group (MS vs. Healthy Middle vs. Healthy Young) and within-subject factors Session and Blocks [Retest20min (S9 vs. S10) vs. Retest24 hours (S13 vs. S14); Figure 2]. The analysis disclosed a main Group effect $\left(F_{(2,66)}=24.6\right.$; $p<0.001 ; M S E=57884 ;$ partial $\left.-\eta^{2}=.43\right)$. MS patients were globally slower that Healthy Middle and Healthy Young controls (Tukey post-hoc $p s<.001$ ), and Healthy Middle slower than Healthy Young $(\mathrm{p}=.03)$. The Age covariate modulated RT performance $\left(F_{(1,66)}=31 ; p=.001 ; M S E=\right.$ 
57884; partial $-\eta^{2}=.32$ ) but did not interact with the main factors Group, Session or Blocks ( $p s>$ .19). The Group $\times$ Session interaction did not reach significance (trend; $F_{(1,66)}=2.6 ; p=.08$; $M S E=4492 ;$ partial $\left.-\eta^{2}=.07\right)$ but the Session $\times$ Block $\times$ PSQI was significant $-\left(F_{(1,66)}=3.9 ; p=\right.$ $.05 ; M S E=3545$; partial $-\eta^{2}=.04$ ), suggesting that sleep quality as assessed by PSQI scores differentially modulated the evolution of performance across groups.

\subsubsection{Offline evolution of sequential learning}

The learning of sequential regularities and its evolution with time were assessed looking at transfer effects from sequential to random blocks, computed using the Interference index (Figure 3). A repeated-measure ANOVA on the Interference index with within-subject factor Session (Learning vs. Retest20min vs. Retest24hours) and between-subject factor Group (MS vs. Healthy Middle vs. Healthy Young $)$ disclosed a main $\operatorname{Group}\left(F_{(2,66)}=4.77 ; p=.01 ; M S E=176 ;\right.$ partial $\left.-\eta^{2}=.13\right)$ as well as a significant Group X Session $\left(F_{(4,132)}=6.4 ; p<.001 ; M S E=43.39 ;\right.$ partial $\left.-\eta^{2}=.16\right)$ effects. The Session effect was non-significant but with a trend $\left(F_{(2,132)}=2.7 . ; p=.067 ; M S E=\right.$ 43.39; partial- $\left.\eta^{2}=.04\right)$. Tukey post-hoc tests conducted on the main Group effect indicated a globally lower Interference index for patients with MS (16.6 \pm 8.5$)$ than Healthy Middle (22.7 \pm $10.8 ; p<.05)$ or Healthy Young $(24.9 \pm 9 ; p<.001$; Healthy Middle $=$ Healthy Young; $\mathrm{p}>.65)$ controls.

Regarding the Group $\times$ Interference index interaction, Tukey post-hoc tests indicate that Healthy Young exhibited an increased interference effect between the Learning and the Retest20min $(p<.005)$ and the Retest20min and the Retest24h $(p<.03)$ sessions, whereas this evolution was not significant neither in MS patients nor Healthy Middle (Learning vs. Retest20min; $p s>.1$; Restest20min vs. Retest24h; ps >. 23). When looking at between-group differences in each session, the interference effect did not differ between groups in the Learning and Retest20min (all $p s>$. 38), but the interference effect in the Restest24h session was lower in MS patients than in both Healthy Young $(p<.001)$ and Healthy Middle $(p<.005)$. The interference effect was not different between Healthy Young and Middle at Restest24h ( $p>.78$; Figure 3).

\section{INSERT FIGURE 3}




\subsection{Sleep and Fatigue scores}

In a second step, we investigated whether self-reported sleep and fatigue measures relate to motor learning and consolidation. Pearson correlation analyses investigating the association between sleep quality (PSQI) and differential interference index scores (i.e., Interference Retest $24 \mathrm{~h}$ minus Retest20min) did not disclose any significant correlation $(p=.6, r=-0.05)$.

\section{DISCUSSION:}

In the present study, we investigated the acquisition of motor sequential material and its evolution with time in patients with MS as several knowledge gaps still exists (for a review, see e.g. (19)). At the motor level, despite a global slowing down in processing speed, we evidence unimpaired learning and overnight consolidation in MS. However, we also observe a decreased sensitivity to interference at delayed overnight testing in patients as compared to heathy controls, suggesting that long-term consolidation of sequential knowledge is impaired in MS disease.

At the motor level, slower RTs in MS patients than in healthy young and middle-aged controls is in line with previous reports $(4,20)$. Reduced motor speed may be explained in part by MS-related physical disabilities (EDSS > 3.5), but may also likely be a reflection of a general cognitive processing slowdown with the evolution of MS disease (21). Additionally, motor learning slopes and interference effects were similar in controls and patients during the learning phase and 20 minutes later, which is also in line with previous studies indicating a preserved capacity to acquire motor sequential knowledge in MS, at least in patients with minimal disability (EDSS >2) (18). It remains to be ascertained how motor and sequential learning capabilities are impacted in the context of the progression of neurological impairments in the course of MS pathology, besides basic motor restrictions.

Sequential and motor learning and their further consolidation take place across different time steps that rely on partially different neuronal networks. Whereas cortico-cerebellar and cortico-striatal loops are recruited during the initial motor learning phase, later steps and especially the acquisition of sequential motor regularities are mainly subtended by coordinated activity between cortical motor areas and subcortical structures including the basal ganglia (22). It was proposed that striatal dysfunction may decrease communication with cortical structures (3), which 
in turn would affect motor acquisition in the early stages of learning (23). At the delayed consolidation stage, post-training sleep-related consolidation effects involve a preferential recruitment of cortico-striatal networks (24), as well as reactivation of connectivity with the basal ganglia during the REM sleep period (25). Besides REM sleep, NREM2 sleep spindles activity was shown involved in the consolidation of sequential motor skills by temporarily synchronizing cortical and subcortical networks including thalamus and striatum $(12,26)$. Since these subcortical structures undergo microstructural changes in the course of MS due to grey matter damage, and knowing that these changes impact on motor performance (3), it should eventually lead to a reduced long-term consolidation efficiency, that we observe here for the sequential but not the motor component of motor sequence learning.

Studies conducted with patients suffering from Parkinson's disease (PD), a pathology associated with basal ganglia neurodegeneration, further highlight a striatal involvement in motor memory consolidation. Indeed, striatum functions strongly rely on the adequate transmission of dopamine (27), and striatal dopamine imbalance is associated with well documented motor impairments in PD, as well as with procedural memory (28) and motor acquisition and consolidation (29) deficits. Furthermore, dopaminergic dysregulation is known to be involved in the occurrence of mental fatigue (30), a phenomenon that affects about $80 \%$ of patients with MS (31). This suggests a possible involvement of fatigue together with altered sleep and cerebral activity (5) in impaired sequential motor memory consolidation, subtended by alterations in the cortico-striatal networks. We conducted an additional analysis (Supplemental material) to investigate this putative relationship, showing that patients with MS with higher scores of mental fatigue obtain significantly lower overnight interference effects, suggesting a possibly jointly altered neuronal network subtending fatigue and motor-learning in MS disease. Results remained significant when considering sleep quality as a potentially confounding covariate. Alternatively, a metabolic explanation can also be considered, dopaminergic unbalance possibly subtending motor off-line consolidation difficulties (29). In this way, the more fatigued the patients are, the more depleted their dopaminergic system would be, and the less consolidated their motor sequence learning experience. Dedicated neuroimaging protocols should investigate further this issue.

In the present study, patients with MS achieved similar motor learning performance levels than controls. They also exhibited similar interference effects after presentation of a random sequence during the learning and 20-minutes delay phase, which confirms their ability to acquire 
sequential regularities. Although performance level $20 \mathrm{~min}$ post-training was similar to the level achieved 24 later in heathy participants, in line with prior studies $(8,9)$, interference effects reflecting the consolidation of sequential knowledge stabilized or increased in healthy control participants only. The more stable is the consolidation of an acquired sequence, the greater is the interference caused by the presentation of a novel sequence $(11,32)$. However, such effect was not observed in MS patients whose interference effect actually decreased overnight as compared to both control groups, independently of age. Although it is tempting to associate impaired motor sequence learning consolidation in patients with MS to their decreased sleep quality (33), the absence of a sleep deprivation control condition (11) in the present experiment does not allow disentangling time- from sleep-dependent effects on impaired memory consolidation in MS disease. At this stage, we conclude that MS patients exhibit impaired consolidation of sequential knowledge 24-hours post-training including a sleep period.

To sum up, this study highlights differential motor memory consolidation deficits in MS disease. Patients with MS exhibited a preserved capacity to acquire and consolidate motor and sequential learning skills in the short-term, but not after a $24 \mathrm{~h}$-period. This suggest that time (or possibly sleep) was not beneficial to consolidate sequential motor skills in patients with MS. Therefore, our results challenge the idea that motor learning is completely preserved in MS disease. Considering that preservation of neuronal plasticity in MS is a key assumption for many reeducation protocols, and particularly motor rehabilitation $(34,35)$, it calls for further research linking motor memory consolidation, mental fatigue and activity in cortico-striatal networks.

\section{Acknowledgements}

GB was supported by the Program P7/33 IPA/PAI COOL. CEB was supported in part by the Reg and Molly Buck award. PP is Francqui Research Professor 2013-2016. The Authors declare that there is no conflict of interest.

\section{References}

1. Horakova D, Kalincik T, Blahova Dusankova J, Dolezal O. Clinical correlates of grey matter pathology in multiple sclerosis. BMC Neurology. 2012. 
2. Leocani L, Comi E, Annovazzi P, Rovaris M, Rossi P, Cursi M, et al. Impaired short-term motor learning in multiple sclerosis: Evidence from virtual reality. Neurorehabil Neural Repair. 2007;21(3):273-8.

3. Cavallari M, Ceccarelli A, Wang G-Y, Moscufo N, Hannoun S, Matulis CR, et al. Microstructural changes in the striatum and their impact on motor and neuropsychological performance in patients with multiple sclerosis. PLoS One. 2014;9(7):e101199.

4. Tomassini V, Johansen-Berg H, Leonardi L, Paixao L, Jbabdi S, Palace J, et al. Preservation of motor skill learning in patients with multiple sclerosis. Mult Scler J. 2011;17(1):103-15.

5. Borragán G, Gilson M, Atas A, Slama H, Lysandropoulos A, De Schepper M, et al. Cognitive fatigue, sleep and cortical activity in multiple sclerosis disease. A Behavioral, Polysomnographic and Functional Near-Infrared Spectroscopy Investigation. Front Hum Neurosci. 2018;

6. Schwarb H, Schumacher EH. Generalized lessons about sequence learning from the study of the serial reaction time task. Advances in Cognitive Psychology. 2012.

7. Karni A, Meyer G, Rey-Hipolito C, Jezzard P, Adams MM, Turner R, et al. The acquisition of skilled motor performance: Fast and slow experience-driven changes in primary motor cortex. Proc Natl Acad Sci U S A. 1998;

8. Hotermans C, Peigneux P, de Noordhout AM, Moonen G, Maquet P. Repetitive transcranial magnetic stimulation over the primary motor cortex disrupts early boost but not delayed gains in performance in motor sequence learning. Eur J Neurosci. 2008 Sep;28(6):1216-21.

9. Hotermans C, Peigneux P, Maertens de Noordhout A, Moonen G, Maquet P. Early boost and slow consolidation in motor skill learning. Learn Mem. 2006;13(5):580-3.

10. Albouy G, Ruby P, Phillips C, Luxen A, Peigneux P, Maquet P. Implicit oculomotor sequence learning in humans: Time course of offline processing. Brain Res. 2006;

11. Borragán G, Urbain C, Schmitz R, Mary A, Peigneux P. Sleep and memory consolidation: Motor performance and proactive interference effects in sequence learning. Brain Cogn. 2015;95:54-61.

12. Boutin A, Doyon J. A sleep spindle framework for motor memory consolidation. Philosophical Transactions of the Royal Society B: Biological Sciences. 2020.

13. Janacsek K, Fiser J, Nemeth D. The best time to acquire new skills: Age-related differences in implicit sequence learning across the human lifespan. Dev Sci. 2012;

14. Buysse DJ, Reynolds CF, Monk TH, Berman SR, Kupfer DJ. The Pittsburgh sleep quality index: A new instrument for psychiatric practice and research. Psychiatry Res. 1989;

15. Penner IK, Raselli C, Stöcklin M, Opwis K, Kappos L, Calabrese P. The Fatigue Scale for Motor and Cognitive Functions (FSMC): Validation of a new instrument to assess multiple sclerosis-related fatigue. Mult Scler. 2009;

16. Kurtzke JF. Rating neurologic impairment in multiple sclerosis: An expanded disability 
status scale (EDSS). Neurology. 1983;

17. Ellis BW, Johns MW, Lancaster R, Raptopoulos P, Angelopoulos N, Priest RG. The St Mary’s Hospital Sleep Questionnaire : A Study of Reliability. Sleep. 1981;4(1):93-7.

18. Monk TH. The post-lunch dip in performance. Clinics in Sports Medicine. 2005.

19. Tablerion JM, Wood TA, Hsieh KL, Bishnoi A, Sun R, Hernandez M, et al. Motor Learning in People with Multiple Sclerosis: A Systematic Review and Meta-analysis. Archives of Physical Medicine and Rehabilitation. 2020.

20. Tacchino A, Bove M, Roccatagliata L, Luigi Mancardi G, Uccelli A, Bonzano L. Selective impairments of motor sequence learning in multiple sclerosis patients with minimal disability. Brain Res. 2014;1585:91-8.

21. Guimarães J, Sá MJ. Cognitive dysfunction in Multiple Sclerosis. Front Neurol. 2012;

22. Doyon J, Benali H. Reorganization and plasticity in the adult brain during learning of motor skills. Curr Opin Neurobiol. 2005 Apr;15(2):161-7.

23. Laforce R, Doyon J. Distinct contribution of the striatum and cerebellum to motor learning. Brain Cogn. 2001;45(2):189-211.

24. Debas K, Carrier J, Barakat M, Marrelec G, Bellec P, Hadj Tahar A, et al. Off-line consolidation of motor sequence learning results in greater integration within a corticostriatal functional network. Neuroimage. 2014 Oct;99:50-8.

25. Peigneux P, Laureys S, Fuchs S, Destrebecqz A, Collette F, Delbeuck X, et al. Learned material content and acquisition level modulate cerebral reactivation during posttraining rapid-eye-movements sleep. Neuroimage. 2003 Sep;20(1):125-34.

26. Boutin A, Pinsard B, Boré A, Carrier J, Fogel SM, Doyon J. Transient synchronization of hippocampo-striato-thalamo-cortical networks during sleep spindle oscillations induces motor memory consolidation. Neuroimage. 2018;

27. Sommer WH, Costa RM, Hansson AC. Dopamine systems adaptation during acquisition and consolidation of a skill. Front Integr Neurosci. 2014;8(November).

28. Nicastro N, Manuel AL, Garibotto V, Burkhard PR, Schnider A. Consolidation of a Learned Skill Correlates with Dopamine SPECT Uptake in Early Parkinson's Disease. J Clin Neurol. 2018;14(4):505-12.

29. Kawashima S, Ueki Y, Kato T, Ito K, Matsukawa N. Reduced striatal dopamine release during motor skill acquisition in Parkinson's disease. PLoS One. 2018;

30. Dobryakova E, Genova HM, DeLuca J, Wylie GR. The dopamine imbalance hypothesis of fatigue in multiple sclerosis and other neurological disorders. Vol. 6, Frontiers in Neurology. 2015.

31. Lerdal A, Gulowsen Celius E, Krupp L, Dahl AA. A prospective study of patterns of fatigue in multiple sclerosis. Eur J Neurol. 2007;

32. Krakauer JW, Ghez C, Ghilardi MF. Adaptation to visuomotor transformations: 
consolidation, interference, and forgetting. J Neurosci. 2005 Jan;25(2):473-8.

33. Buratti L, Iacobucci DE, Viticchi G, Falsetti L, Lattanzi S, Pulcini A, et al. Sleep quality can influence the outcome of patients with multiple sclerosis. Sleep Med [Internet]. 2019;58:56-60. Available from:

https://www.sciencedirect.com/science/article/pii/S1389945719300607

34. Lipp I, Tomassini V. Neuroplasticity and motor rehabilitation in multiple sclerosis. Front Neurol. 2015;6(MAR).

35. Ghai S, Ghai I. Effects of rhythmic auditory cueing in gait rehabilitation for multiple sclerosis: A mini systematic review and meta-analysis. Frontiers in Neurology. 2018. 
a)

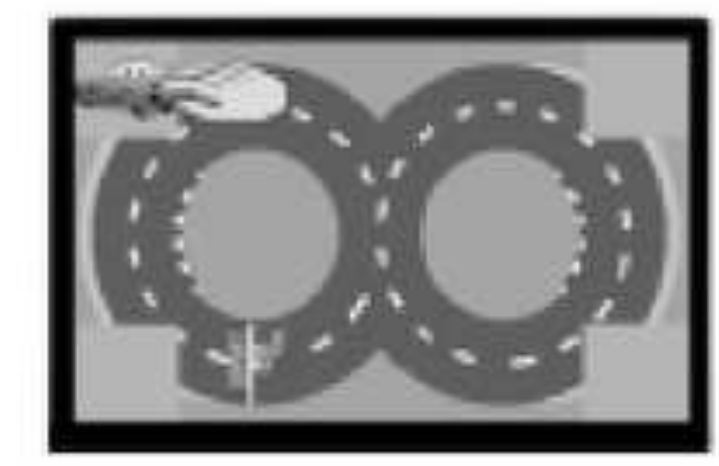

Day 1 timeline

b)

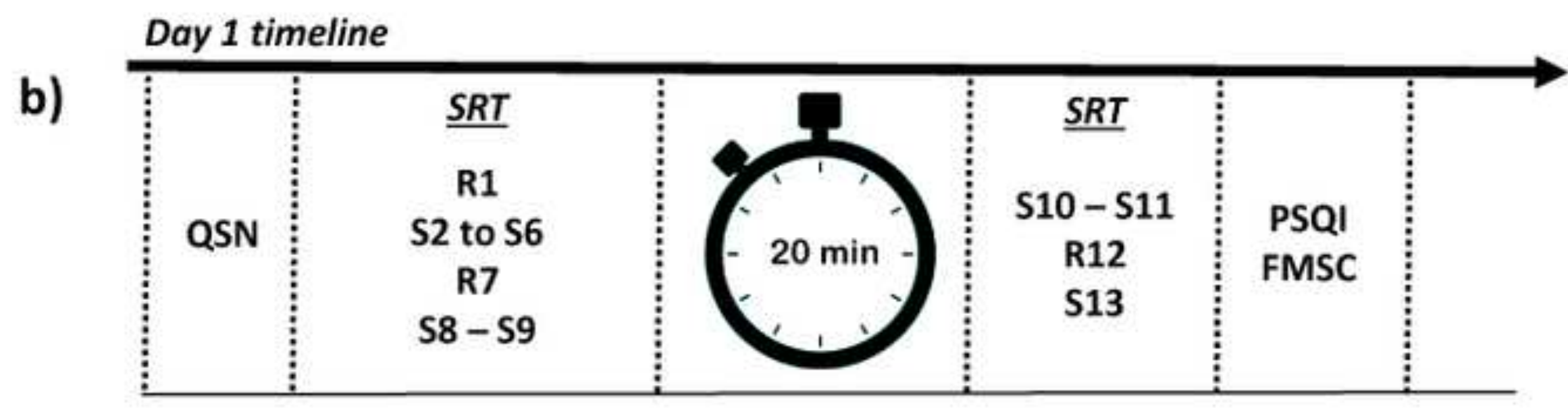

Day 2 timeline

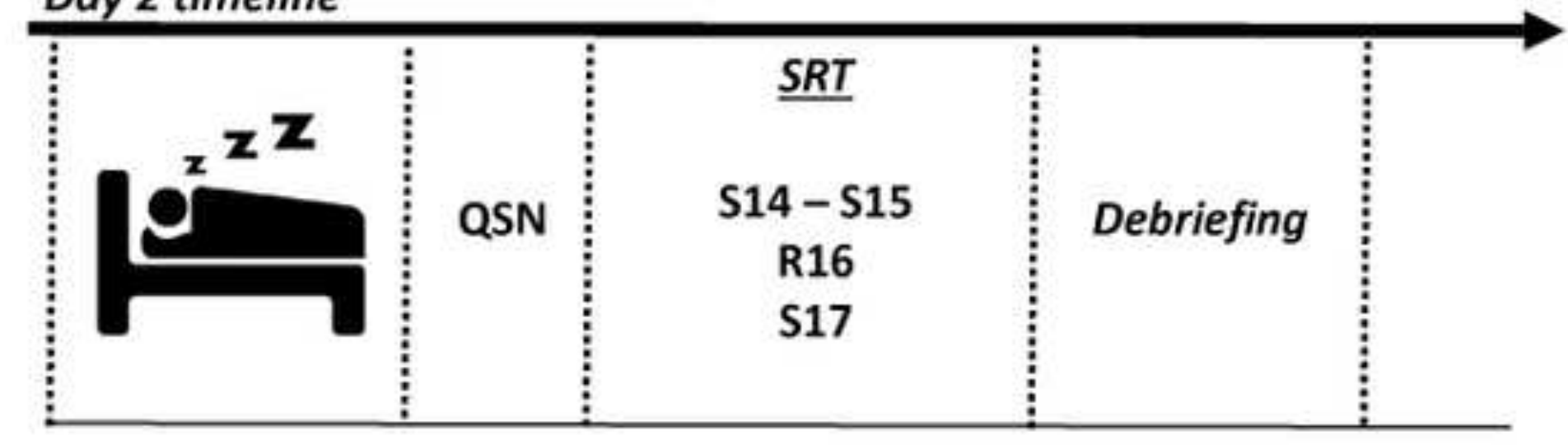




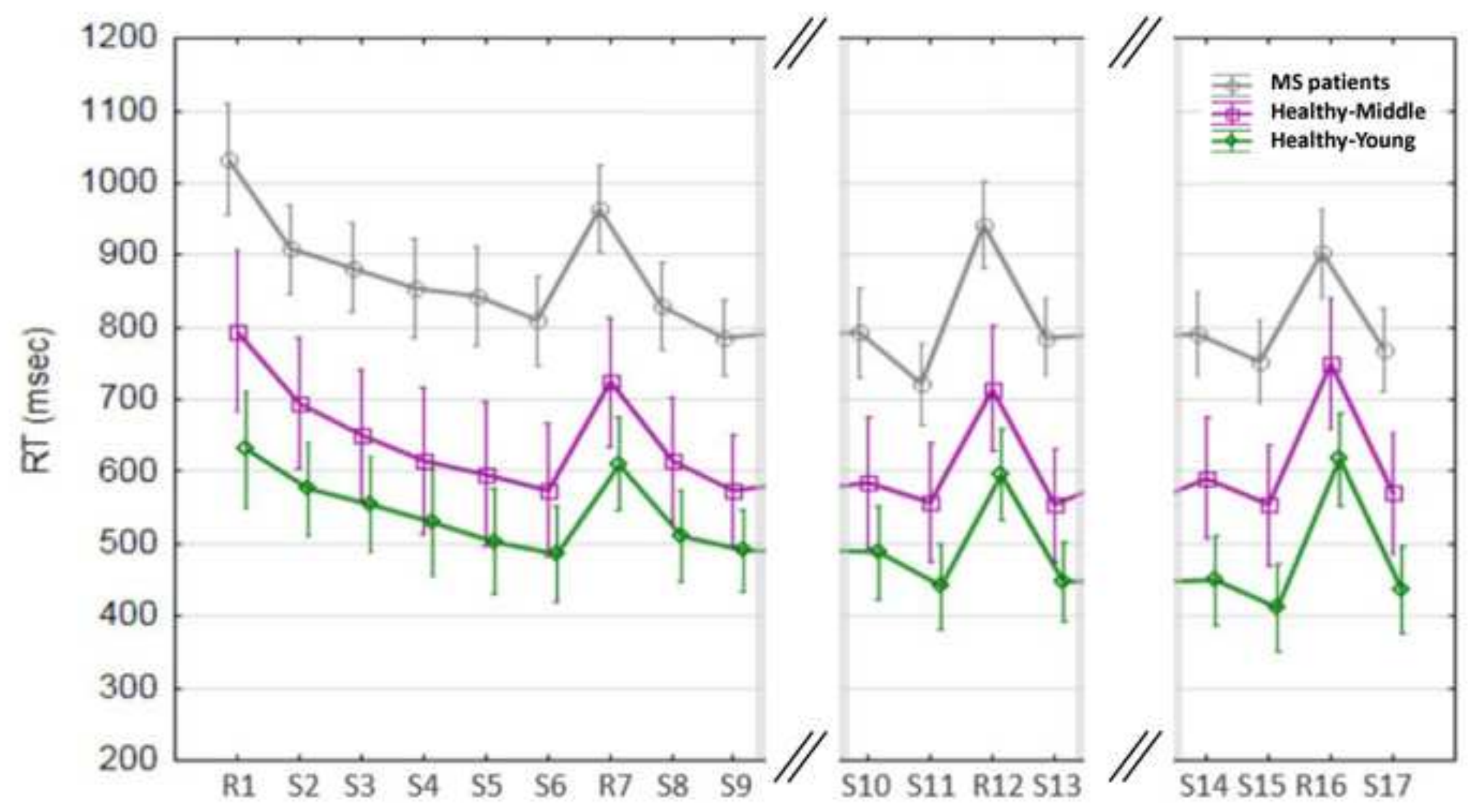




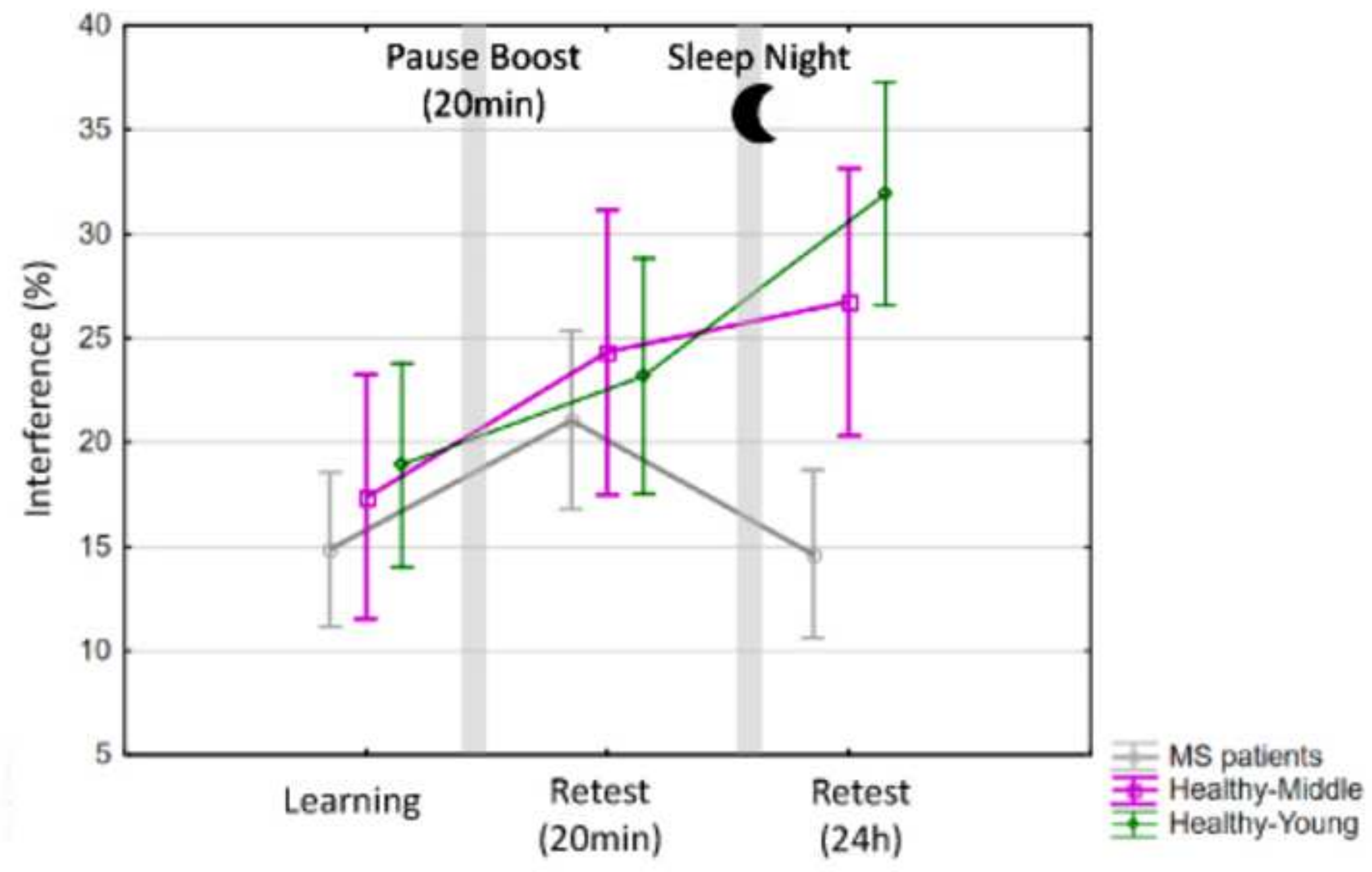


Figure 1: a) Stimulus (car picture) presentation at one of the four possible corner locations on the touchscreen. b) Overview of the experimental procedure. SRT = Serial Reaction Time task: blocks with prefix "S" $(2-6,8,9,10-11,13,14-15,17)$ repeat 8 times the learned 8 -elements sequence, blocks with prefix “R” $(1,7,12,16)$ present 64 random locations; PSQI = Pittsburgh Sleep Quality Index; FSMC = Fatigue Scale for Motor and Cognitive functions; QSN = St-Mary Hospital Sleep Questionnaire.

Figure 2. SRT Performance. Mean +/- SEM reaction times (msec) per block in MS, Middle-Age and Young adult participants in the learning (R1, S2-S6, R7, S8-S9), 20-minutes (S10-S11, R12, S13) and 24-hours (S14-S15, R16, S17) sessions.

Figure 3: Evolution of transfer effects (Interference index) reflecting the acquisition of sequential regularities across sessions. Computed with co-variates at their means for ages $(M=37.26)$ and sleep quality (PSQI: $M=6.86$ ). 
Table 1: Age, Sleep and Fatigue

\begin{tabular}{lcccc}
\hline & Patients with MS & $\begin{array}{c}\text { Healthy } \\
\text { Middle }\end{array}$ & $\begin{array}{c}\text { Healthy } \\
\text { Young }\end{array}$ \\
\cline { 2 - 5 } & & 30 & 14 & 27 \\
\cline { 2 - 5 } Age (years) & Mean \pm SD & Mean \pm SD & Mean \pm SD \\
\cline { 2 - 5 } PSQI & $44.5 \pm 14.20$ & $53.1 \pm 5.8^{*}$ & $21.00 \pm 1.20^{*}$ \\
EDSS & $8.90 \pm 4.3$ & $5.30 \pm 2.00^{*}$ & $5.40 \pm 2.20^{*}$ \\
FSMC & Physical & $29.8 \pm 10.1$ & $/$ & $/$ \\
& Cognitive & $30.5 \pm 10$ & $/$ & $/$ \\
& Psychosocial & $6.3 \pm 2.6$ & $/$ & $/$
\end{tabular}

Notes. PSQI = Pittsburgh Sleep Quality Index, global score; EDSS = Expanded Disability Status Scale ; FSMC = Fatigue Scale for Motor and Cognitive functions; $M$. = Mean; SD = Standard deviation. ${ }^{*}$ Tukey post-hoc significant difference $p<.05$ with regard to patients with MS 


\section{The effect of cognitive fatigue for motor learning in MS patients}

In a complementary analysis, we aimed at investigating the potential effect of cognitive fatigue on motor learning in MS disease. To compare whether the presence of fatigue influenced motor learning, we tentatively subdivided MS patients into two groups according to the severity of their fatigue level as evaluated using the FSMC (1). Patients were differentiated between Severe (FSMC-cognitive $>27 ; \mathrm{N}=15$ ) and Mild (FSMC-cognitive $<27 ; \mathrm{N}=15$ ) cognitive fatigue levels. A one-tailed Mann-Whitney independent sample test did not evidence significant differences between Severe and Mild conditions regarding Age and the Expanded Disability Status Scale - EDSS (2) (all ps $>$.2). A trend for worse usual sleep quality was observed in MS patients with Severe fatigue $(\mathrm{p}=0.07$; see Supplementary Table 1). As expected, given the categorization criteria, FSMC-cognitive and physical scores were higher in MS patients with Severe than Mild fatigue.

\section{$\underline{\text { Supplementary Table } 1}$}

\begin{tabular}{lcc}
\hline Dimensions & Mild fatigue & Severe fatigue \\
\hline $\mathbf{N}$ & 15 & 15 \\
\cline { 2 - 3 } & $\mathbf{M}$. / SD & M. / SD \\
\cline { 2 - 3 } Age & $42.667 / 15.141$ & $46.400 / 13.405$ \\
EDSS & $3.700 / 3.104$ & $3.733 / 3.052$ \\
PSQI & $7.600 / 4.205$ & $10.133^{\mathrm{f}} / 4.051$ \\
FSMC Cognitive fatigue & $22.267 / 5.431$ & $37.933^{* * *} / 7.146$ \\
FSMC Physical fatigue & $24.800 / 10.469$ & $37.000^{* * *} / 4.943$ \\
\hline Notes. M. = Mean ; SD = Standard deviation ; one-tailed Mann-Whitney t-tests ${ }^{* * *}=\mathrm{p} \leq .001{ }^{\mathrm{f}} \mathrm{p}=0.07$
\end{tabular}




\section{Motor learning in MS patients with Mild vs. Severe fatigue}

A repeated-measure ANOVA with within-factor Blocks (R1-S6) and between-factor cognitive fatigue Subgroups (Severe vs. Mild) was computed to investigate potential differences in the evolution of learning. The analysis disclosed a main Block effect $\left(F_{(5,140)}=14 ; p<.001 ; M S E=\right.$ 13197; partial $\left.-\eta^{2}=.33\right)$, but no main Subgroup $(p>.28)$ or interaction $(p>.27)$ effects. Similarly, there was no evidence for a motor performance enhancement (i.e., boost effect) after a short break (S9-S10; all ps >0.26).

Finally, we investigated the sensitivity of MS patients with Mild vs. Severe fatigue to the introduction of the transfer block (Interference index), i.e., sequence learning. A repeatedmeasure ANOVA with within-subject factor Interference index (Learning vs. Retest20min vs. Retest24hours) and between-subject factor Subgroup (Severe vs. Mild) disclosed a main Subgroup $\left(F_{(1,28)}=4.3 ; p<.05 ; M S E=99.7 ;\right.$ partial $\left.-\eta^{2}=.13\right)$ effect with a lower interference index in MS patients with Severe cognitive fatigue (14.4 \pm 5.8$)$ than with Mild fatigue (18.8 \pm 5.8). The main effect of Interference index was also significant $\left(F_{(2,56)}=4.2 ; p<.05 ; M S E=\right.$ 54.1; partial- $\eta^{2}=.13$ ). Tukey post-hoc revealed higher interference values during the Retest20min $(19.7 \pm 6.1)$ than during the Learning $(14.7 \pm 5.9 ; p<.05)$ and Retest24hours $(15.4$ $\pm 5.6 ; p<.07)$ periods. The Subgroup $\times$ Interference interaction did not reach significance $(p>$ $.27)$

\section{Impact of sleep quality on motor learning in MS patients with Mild vs. Severe fatigue}

To investigate whether subjective sleep quality (as reflected by the Pittsburgh Sleep Quality Index - PSQI (3) global score) contributes to the between-subgroup differences reported above, a repeated-measure ANOVA with within-subject factor Interference index (Learning vs. Retest20min vs. Retest24hours) and between-subject factor Subgroup (Severe vs. Mild) was computed with the global PSQI score entered as a covariate. Although there was a trend for PSQI scores to modulate the amplitude of interference effects $\left(F_{(2,54)}=3 ; p=.056 ; M S E=\right.$ 
50.4 ; partial $\left.-\eta^{2}=.10\right)$, the main Interference effect remained significant $\left(F_{(2,54)}=5 ; p<.02\right.$; $M S E=50.4 ;$ partial $-\eta^{2}=.16$ ) with higher interference values during the Retest20min than the Learning and Retest24hours periods. The main Subgroup (Severe vs. Mild fatigue) effect was no longer significant $\left(F_{(1,27)}=3 ; p=.09 ; M S E=101.1\right.$; partial $\left.-\eta^{2}=.1\right)$, suggesting that the trend for a worse sleep quality in MS patients with Severe fatigue $(\mathrm{p}=0.07$; see Supplementary Table 1 above) may partly contribute to motor learning deficits.

1. Penner IK, Raselli C, Stöcklin M, Opwis K, Kappos L, Calabrese P. The Fatigue Scale for Motor and Cognitive Functions (FSMC): Validation of a new instrument to assess multiple sclerosis-related fatigue. Mult Scler. 2009;

2. Kurtzke JF. Rating neurologic impairment in multiple sclerosis: An expanded disability status scale (EDSS). Neurology. 1983;

3. Buysse DJ, Reynolds CF, Monk TH, Berman SR, Kupfer DJ. The Pittsburgh sleep quality index: A new instrument for psychiatric practice and research. Psychiatry Res. 1989; 